\title{
Open
}

\section{Genotype-phenotype correlation and mutation spectrum in a large cohort of patients with inherited retinal dystrophy revealed by next-generation sequencing}

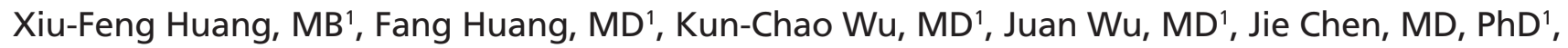 \\ Chi-Pui Pang, PhD², Fan Lu, MD, OD ${ }^{1}$, Jia Qu, MD ${ }^{1}$ and Zi-Bing Jin, MD, PhD ${ }^{1}$
}

Purpose: Inherited retinal dystrophy (IRD) is a leading cause of blindness worldwide. Because of extreme genetic heterogeneity, the etiology and genotypic spectrum of IRD have not been clearly defined, and there is limited information on genotype-phenotype correlations. The purpose of this study was to elucidate the mutational spectrum and genotype-phenotype correlations of IRD.

Methods: We developed a targeted panel of 164 known retinal disease genes, 88 candidate genes, and 32 retina-abundant microRNAs, used for exome sequencing. A total of 179 Chinese families with IRD were recruited.

Results: In 99 unrelated patients, a total of 124 mutations in known retinal disease genes were identified, including 79 novel mutations (detection rate, 55.3\%). Moreover, novel genotype-phenotype correlations were discovered, and phenotypic trends noted. Three cases are reported, including the identification of AHI1 as a novel candidate gene for nonsyndromic retinitis pigmentosa.

Conclusion: This study revealed novel genotype-phenotype correlations, including a novel candidate gene, and identified 124 genetic defects within a cohort with IRD. The identification of novel genotype-phenotype correlations and the spectrum of mutations greatly enhance the current knowledge of IRD phenotypic and genotypic heterogeneity, which will assist both clinical diagnoses and personalized treatments of IRD patients.

Genet Med advance online publication 6 November 2014

Key Words: exome sequencing; genotype-phenotype correlations; novel mutations; retinal dystrophy

\section{INTRODUCTION}

Inherited retinal dystrophy (IRD) is a class of monogenic, vision-impairing disorders that are caused by retinal degeneration. IRD includes retinitis pigmentosa (RP) and allied diseases. The common manifestation is a pathological change in rod and/or cone photoreceptor cells, which are the specialized and light-sensitive neurons in the retina. ${ }^{1}$ More than 200 disease-causing genes associated with IRD have been identified (RetNet, https://sph.uth.edu/retnet/), demonstrating the extreme genetic heterogeneity of the disease and therefore making molecular diagnosis technically challenging, because it entails the commitment of substantial time and resources. ${ }^{2}$

Next-generation sequencing (NGS) is a powerful new approach that enables the simultaneous screening of multiple genes; it has quickly come to be used extensively in molecular diagnosis by sequencing the exome. ${ }^{3-5}$ Notably, this approach has been used for the development of next-generation molecular diagnosis of hereditary eye disorders. ${ }^{6-8} \mathrm{Abu}-\mathrm{Safieh}$ and colleagues $^{6}$ designed an autozygome-guided screening panel in which they were able to successfully identify IRD in patients with six novel candidate genes. In another study, 163 known IRD genes were screened for by exome sequencing in 179 patients with Leber congenital amaurosis and juvenile RP, identifying a genetic predisposition in $40.2 \%$ (72/179) of the cases. ${ }^{8}$ Further verifying this approach, Chen et al. used targeted sequencing of 189 genes in members of 41 Chinese families with IRD and identified mutations occurring in 14 families. ${ }^{9}$ In pilot studies, we successfully performed molecular diagnoses in patients with Usher syndrome and Bardet-Biedl syndrome by assessing a panel of 144 known genes. ${ }^{7,10}$ Despite success in previous studies, there is still a large proportion of patients with IRD who have unidentified genetic mutations. ${ }^{2}$ In addition, there are few thorough studies of the relationship between specific genotypes and phenotypes.

Because NGS allows for the screening of limited networked genes in addition to genes known to be associated with the onset of IRD, targeted exome sequencing of additional candidate genes is a rapid and cost-effective method of revealing novel disease-causing genes. ${ }^{9} 10$ Many novel or unrecognized genes interact with known targets of IRD. ${ }^{12,13}$ In addition, variations in microRNA (miRNA) expression could lead to a significant abnormality in the retina. ${ }^{14}$ Taken together, this information suggests that networked genes and miRNAs potentially interact with key IRD genes, possibly inducing a disease state. 
In this study, we developed a panel of 164 known genes causing retinal disease, 88 possible candidate genes, and 32 retinal miRNAs for genomic DNA capture. This panel was used in NGS of the exomes of 179 unrelated patients with IRD. We discovered 124 pathological mutations in 99 patients, including 79 novel mutations in previously identified IRD genes. Moreover, several genotype-phenotype correlations were uncovered, including identification of $A H I 1$ as a novel candidate gene for nonsyndromic RP. The identification of novel genotype-phenotype correlations and the mutation spectrum not only greatly enhance knowledge of IRD phenotypic and genotypic heterogeneity but also will assist in both the clinical diagnosis and the development of personalized treatments for IRD.

\section{Study subjects}

\section{MATERIALS AND METHODS}

The study protocol was designed in accordance with the tenets of the Declaration of Helsinki and approved by the Ethics Committee of the Eye Hospital of Wenzhou Medical University. A group of 179 Chinese patients diagnosed with IRD by retina specialists were recruited for this study. Informed consent was obtained from all participants. Patients' initial symptoms and complaints included poor vision or night blindness during their first or second decade of life. All patients received a clinical diagnosis based on clinical eye examinations. A detailed family history was obtained from the probands and/or their relatives. Peripheral blood samples were collected from the affected patients as well as from unaffected relatives.

\section{Design of targeted capture panel}

We developed a panel for targeted DNA capture and sequencing by selecting 164 known IRD-related genes from RetNet and OMIM (Supplementary Table S1 online). The panel also included a cluster of 88 possible candidate genes with expression patterns that were spatiotemporally related to retinal development $^{15,16}$ (Supplementary Table $\mathbf{2} 2$ online) and 32 abundant retinal miRNAs ${ }^{17,18}$ (Supplementary Table S3 online). The targeted capture panel included a total of 252 genes, which comprised 3,376 exons and 615,029-nt coding regions. The panel was constructed using the GenCap custom enrichment kit (MyGenostics, Beijing, China) according to the manufacturer's instructions.

\section{Paneled exome sequencing}

A minimum of $3 \mu \mathrm{g}$ of genomic DNA was extracted from each blood sample and was broken into fragments to construct the DNA library. Biotinylated single-strand DNA capture probes were hybridized in solution with the target library. Streptavidincoated magnetic beads were used to wash, elute, and amplify the DNA, which was then subjected to NGS using an Illumina Solexa HiSeq 2000 sequencer.

\section{In-depth bioinformatics analysis}

Sequence reads acquired from raw data were aligned to the reference human genome (hg19) using the SOAPaligner program (http://soap.genomics.org.cn/). Single-nucleotide polymorphisms were initially identified by the SOAPsnp program (http://soap.genomics.org.cn/) after filtering polymerase chain reaction duplicates using SAMtools (http://samtools. sourceforge.net/). Insertions or deletions were analyzed using BWA (http://bio-bwa.sourceforge.net/) and GATK programs (https://www.broadinstitute.org/gatk/) and then were extracted using the 1000 Genome, dbSNP135, and HapMap databases, as well as an in-house database of 500 exomes. Sequence variants were standardized according to Human Genome Variant Society variation nomenclature (http://www.hgvs.org/mutnomen/) and checked using the Mutalyzer program (http:// www.lovd.nl/mutalyzer/). Missense variants were assessed with PolyPhen-2 and SIFT, as described previously. ${ }^{19}$ The splice-site effect was predicted using the BDGP (http://www.fruitfly.org/ seq_tools/splice.html) and ASSP programs (http://wangcomputing.com/assp/index.html).

\section{Sanger sequencing validation and cosegregation analysis}

All of the candidate mutations were confirmed by direct Sanger sequencing using an ABI 3500 Genetic Analyzer (Applied Biosystems, Carlsbad, CA). Variants, including nonsense or frameshift mutations, were considered mutation candidates, expanding the validation that was performed within kinships to analyze cosegregation.

\section{Patients with IRD}

\section{RESULTS}

The study group included 127 isolated cases (70.9\%) and 34 autosomal recessive cases (19.0\%); the remaining 18 cases $(10.1 \%)$ were either autosomal dominant $(n=12)$ or X-linked $(n=6)$. Diagnoses of participating subjects included RP, conerod dystrophy, Leber congenital amaurosis, Stargardt disease, congenital stationary night blindness, Bietti crystalline dystrophy, and Usher syndrome (Supplementary Table S4 online).

\section{Exome sequencing of paneled genes}

The genomic DNA derived from the 179 patients with IRD was subjected to massive parallel NGS, which covered a panel of 252 genes and 32 miRNA regions. On average, the mean coverage depth was $191.5 \times$ and included $98.2 \%$ of the targeted regions (Figure 1). These results suggested that exome sequencing combined with direct sequencing is sufficient to yield highquality data for further analysis.

\section{Identification of pathogenic mutations in the IRD cohort}

Bioinformatics analysis, direct sequencing confirmation, and familiar validation from 179 patients enrolled in this study revealed pathological mutations in 99 cases (55.3\%) (Table 1). A total of 124 distinct mutations were identified; 79 novel occurrences had not previously been reported. The other 45 mutations have been reported in previous studies (Supplementary Table S5 online). Notably, among the list of 79 novel mutations, $42(53.2 \%)$ were nonsense, frameshift, or splice-site mutations that severely affected protein function; the other 37 novel 


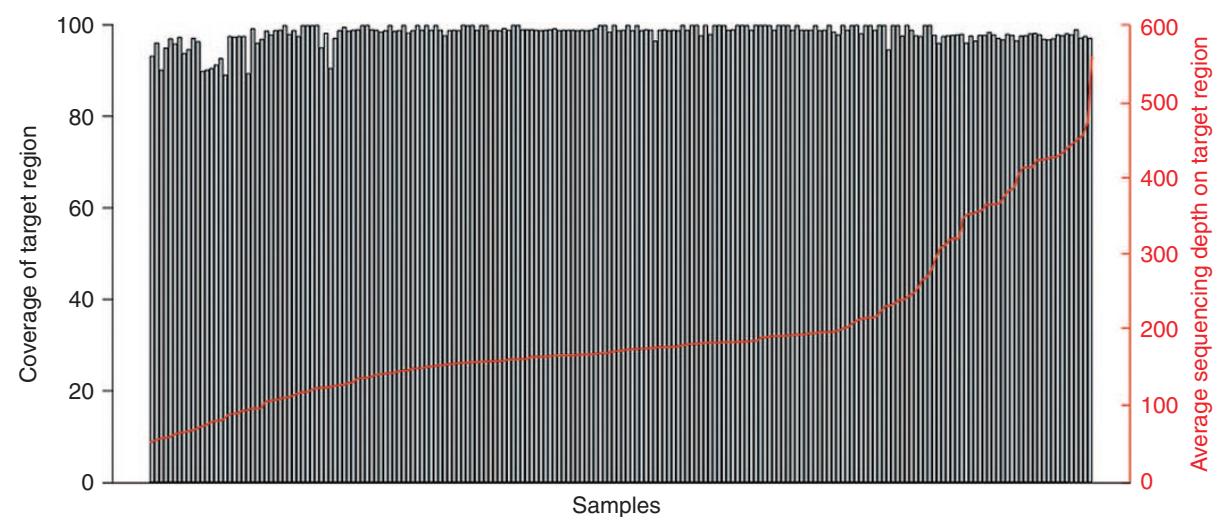

Figure 1 Primary data statistics of deep exome sequencing. The black histogram represents the coverage of the target region in each sample. The red line indicates the average sequencing depth of the target region.

Table 1 Distribution and detection rates in patients with inherited retinal dystrophy

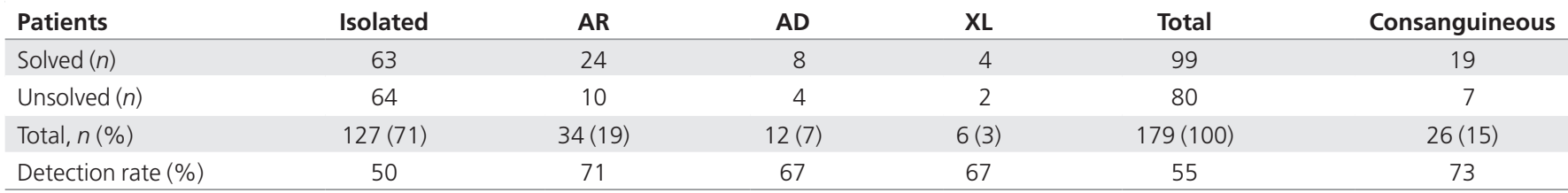

$A D$, autosomal dominant; $A R$, autosomal recessive; $X L, X$ chromosome-linked.

missense mutations (46.8\%) were predicted to have deleterious protein functional effects in silico. All of these mutations were either absent from the control database or their minor allele frequencies were less than 0.01 . Furthermore, cosegregation testing among family members indicated that the mutations cosegregated with disease phenotype. Compound heterozygous mutations or homozygous mutations were confirmed by cosegregation in parents or siblings of affected patients. Dominant mutations either caused multigeneration pedigrees or were confirmed to be de novo mutations. Taken together, these results demonstrated that NGS is a powerful approach for molecular diagnosis of IRD.

\section{Mutation spectrum in Chinese patients with IRD}

Among the 179 probands in this study, 161 of the cases (90\%) were sporadic or recessive, and causative mutations were successfully identified as genetic defects in 99 unrelated families (55.3\%) (Table 1; Supplementary Tables S4 and S5 online). The genotyping data obtained from these patients also indicated a mutation spectrum (Figure 2). Among the 127 patients with sporadic IRD, 63 cases $(50 \%)$ harbored mutations, whereas, intriguingly, 50 others ( $79 \%$ of the total solved sporadic cases) carried mutations in their autosomal recessive genes, with 10 autosomal dominant (16\%) and $3 \mathrm{X}$-chromosome linked (5\%) mutations. These results could be helpful in genetic counseling for patients with sporadic IRD, who are frequently seen in clinics. In this study, 34 families were definite autosomal recessives, whereas 24 were successfully determined to have genetic predispositions, with a detection rate as high as $71 \%$ for autosomal recessive IRD (Table 1; Supplementary Tables S4 and S5 online). Among the 26 consanguineous families, mutations were identified in 19 (73\%). Interestingly, all mutations were in recessive genes (Table 1; Supplementary Tables S4 and S5 online). In our mutation pool, the top three genes contributing to IRD were USH2A, EYS, and CRB1 (Figure 2). These genes with a high frequency of mutations should be a priority during genetic screening of Chinese patients. These results bring to light, for the first time, a mutational spectrum in Chinese patients with IRD, and they provide valuable insights not only for genetic counseling but also for the diagnosis and therapy of patients with IRD.

\section{Screening of possible candidate genes and miRNAs}

To reveal any underlying genetic defect in novel genes, we screened 88 possible candidate genes (Supplementary Table S2 online) and 32 miRNAs (Supplementary Table S3 online). However, no pathological mutations were found in any of these genes or miRNA regions. This result indicated that the selected genes and miRNAs either do not confer a genetic predisposition to IRD or that the incidence is very low in these patients.

\section{Evaluation of genotype-phenotype correlations}

To investigate the relationship between mutated genes and IRD symptoms, we performed genotype-phenotype analyses of all patients with IRD with disease-causing mutations. Clinical data for all 99 patients, including the most recent best-corrected visual acuity, disease duration, and age at onset, are shown in a scatterplot based on 35 different genes (Figures 3 and 4). The results of assessment of visual acuity are represented by different colors. Disease duration was calculated as the difference between current age and age at onset. 


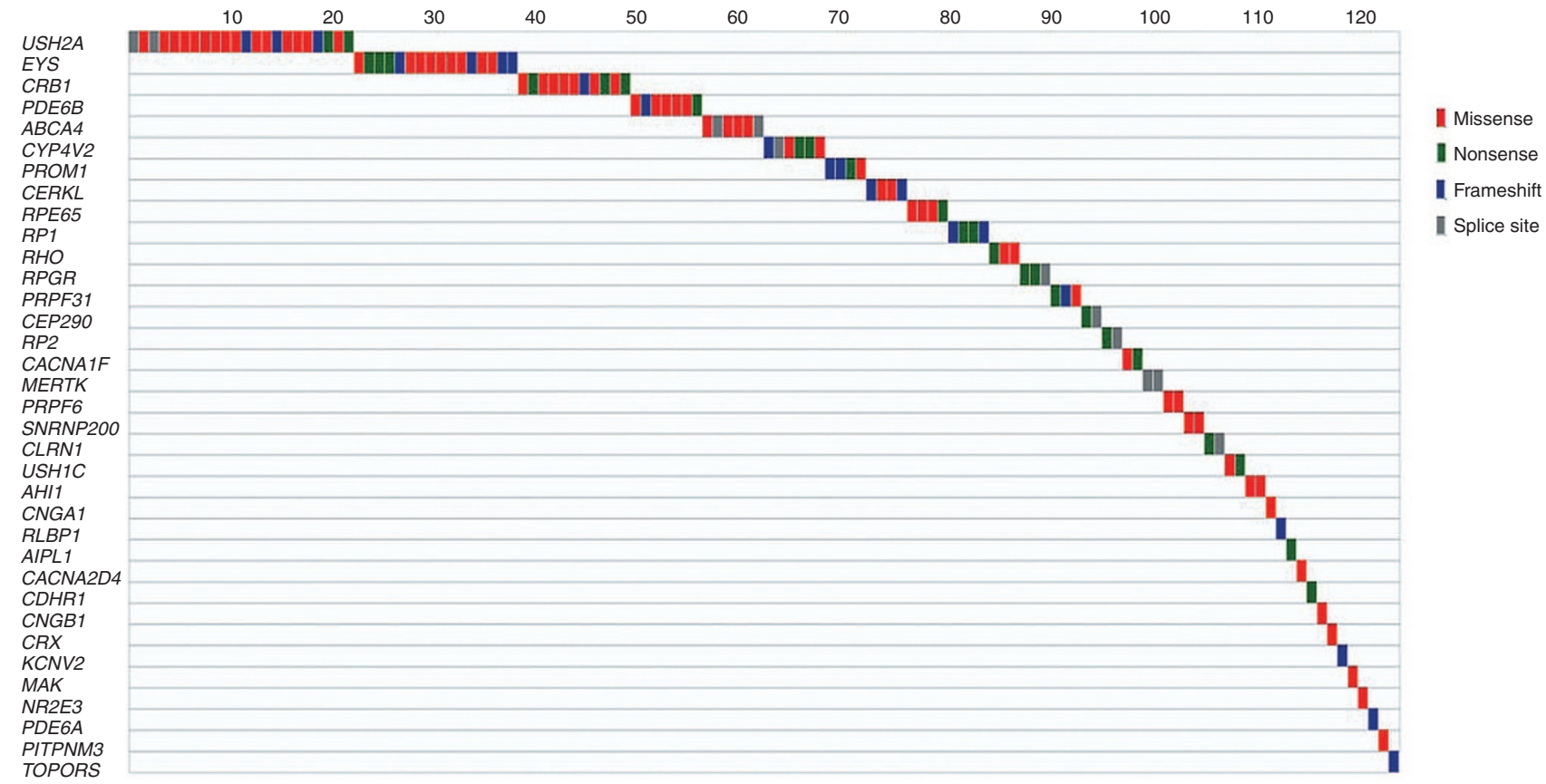

Figure 2 Mutation spectrum in Chinese patients with inherited retinal dystrophy (IRD). The mutation spectrum indicates the range of mutations observed in the Chinese IRD cohort. Each colored box represents a mutation type: red, missense; green, nonsense; blue, frameshift; gray, splice-site. The most frequently mutated genes were USH2A, EYS, and CRB1.

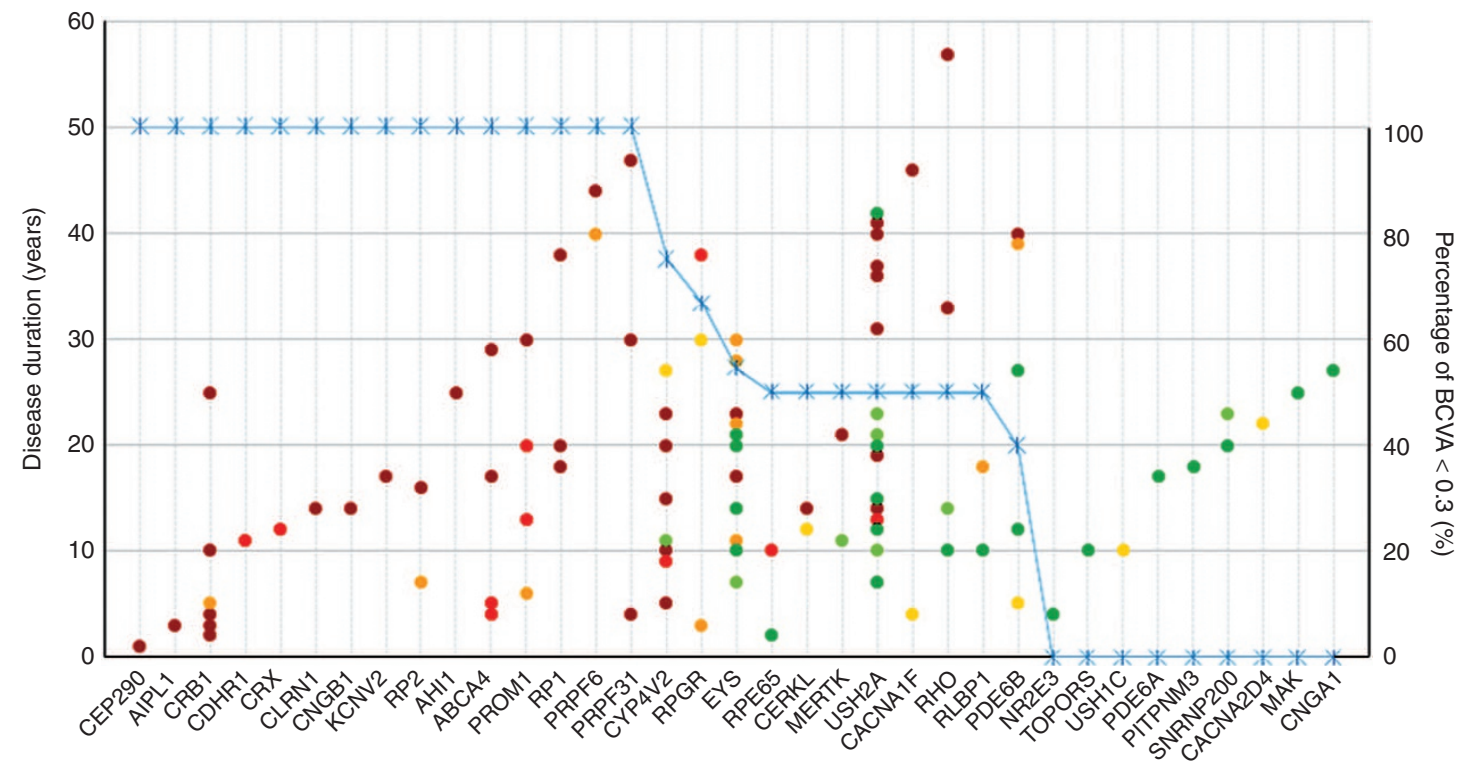

Figure 3 Relationship between visual acuity and specific causative genes. The phenotypic severity of visual acuity is classified into six groups, which are plotted in different colors, according to the causative genes and disease duration. The disease duration is calculated as the difference between the current age and age at onset. The blue line represents the percentage of patients with best-corrected visual acuity (BCVA) less than 0.3.

Herein, the total 35 causative genes that were responsible for IRD in 99 families were ranked according to the percentage of best-corrected visual acuity less than 0.3 (Figure 3 ). The genes exhibited on the left of the scatterplot caused severely decreased vision. In this group, best-corrected visual acuity was reduced to less than 0.03 in a high proportion of patients (Figure 3). By contrast, the genes in the right side of the plot correlated with a mild phenotype; almost all patients with these genes had a visual acuity higher than 0.3 (Figure 3 ). The genes in the middle showed a moderate phenotype, including the two genes with highest frequency of mutations:USH2A and EYS. We were also able to clarify the average age at onset and distribution (Figure 4). The red line represents the average age at onset, and the blue histogram exhibits the number distribution of five age groups. The genes to the left caused more early onset disease compared with those on the right 


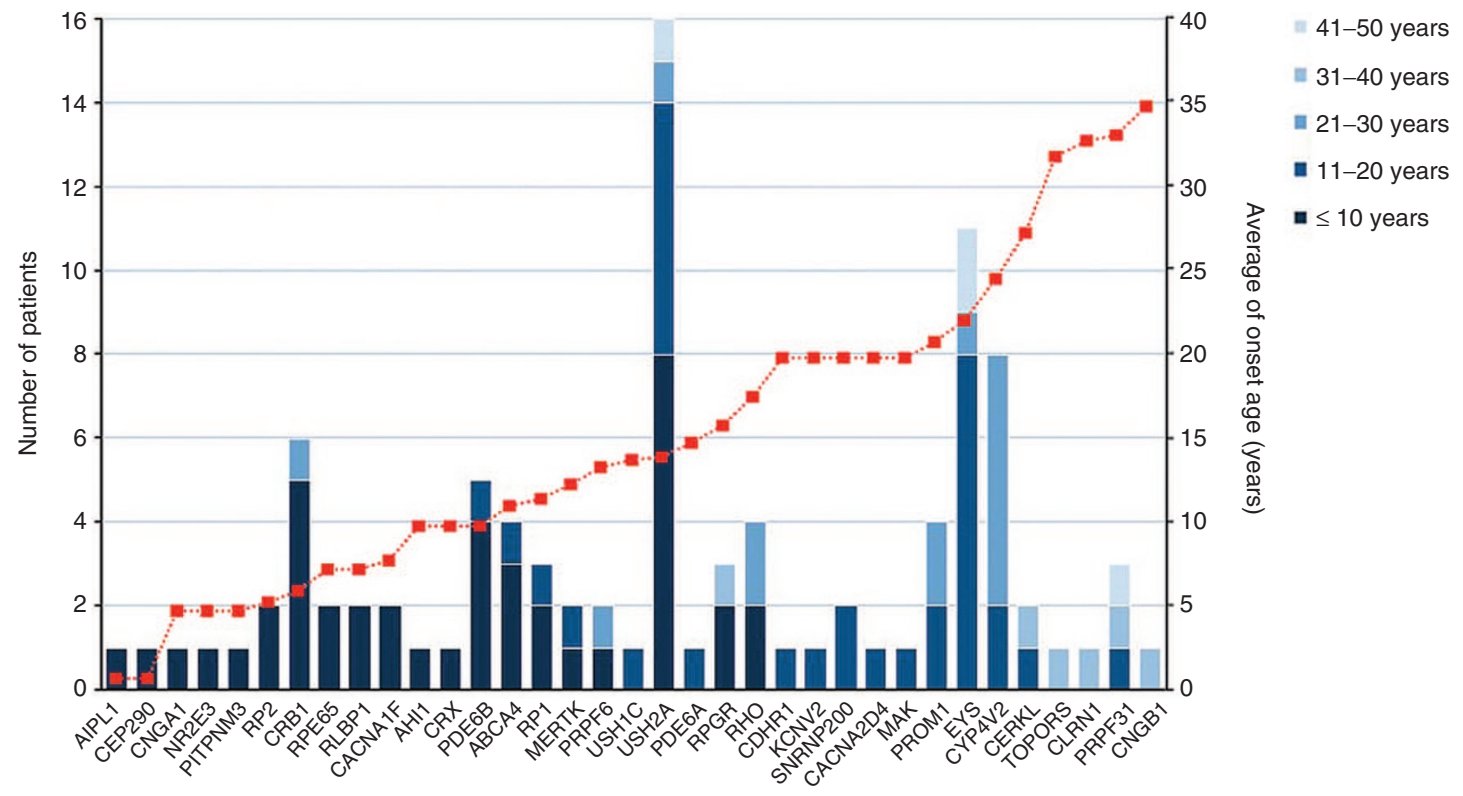

Figure 4 Relationship between average age at onset and specific causative genes. The red line represents the average age at onset, and the blue histogram displays the number distribution of five age groups.

(Figure 4). Despite the high genetic and clinical heterogeneity of IRD, phenotypic trends such as visual acuities and age at onset were correlated with genotypes. Collectively, the results of this study of a large sample bring to light valuable and significant genotype-phenotype correlations.

\section{Revision of the initial clinical diagnosis}

We observed an inconsistency between the initial clinical diagnosis and genetic defects. In a consanguineous family (Supplementary Figure S1 online), patient W29-1 was diagnosed as having sporadic RP. However, a pathogenic homozygous mutation in the NR2E3 gene (c.290G>A, p.R97H) was identified (Supplementary Figure S1 online) that was reported to cause enhanced S cone syndrome (ESCS) in previous studies. ${ }^{20,21}$ To explain this inconsistency, proband W29-1 was sent for a clinical reassessment. Full-field electroretinography showed extinguished rod responses (Supplementary Figure S1 online). The maximal dark-adapted responses had amplitudes significantly below the normal minimum and an unusually broad waveform, and oscillatory potentials were almost absent. After adaptation to light, the cone responses presented abnormal cone waveforms and were similar to the dark-adapted maximal responses. The $30-\mathrm{Hz}$ flicker responses were strikingly weak and delayed (Supplementary Figure S1 online). Electroretinography demonstrated that the phenotype was consistent with typical ESCS. ${ }^{20,21}$ Consequently, we successfully revised the clinical diagnosis of patient W29-1. To the best of our knowledge, this is the first recorded case of ESCS in the Chinese population.

\section{Observation of a novel genotype-phenotype correlation}

In a 67-year-old patient (W83-1) with sporadic RP (Supplementary Figure S2 online), a de novo mutation
(c.628G $>$ T, p.V210F) in the RHO gene was identified, which was reported in a previous study ${ }^{22}$ (Supplementary Figure S2 online). The fundus showed typical bone spicule pigmentation. Interestingly, the macula of the right eye was severely abnormal, showing prominent and deep macular coloboma (Supplementary Figure S2 online). As far as we know, unilateral macular coloboma was first observed in patients with RP with a $R H O$ gene mutation.

\section{Identification of $A H I 1$ as a novel candidate gene for nonsyndromic RP}

AHI1 was previously reported to cause Joubert syndrome (JBS), ${ }^{23}$ a congenital systemic disease associated with the involvement of multiple organs, including the retina, kidneys, skeleton, and liver. In this study we identified compound heterozygous mutations (c.653A $>\mathrm{G}$, p.Y218C; c.3257A $>\mathrm{G}$, p.E1086G) in the AHI1 gene in proband W196-1, who had sporadic RP (Supplementary Figure S3 online). Cosegregation analysis confirmed that the Y218C mutation was inherited paternally and that the E1086G mutation was inherited maternally (Supplementary Figure $\mathbf{3} 3$ online). The missense mutation Y218C was novel, and another mutation was reported previously as one causing JBS. ${ }^{24}$ The novel mutation was predicted in silico to be strongly pathogenic, with an amino acid change in a highly conserved region (Supplementary Figure S3 online). Interestingly, the proband showed only a typical RP phenotype (Supplementary Figure S3 online) without systemic manifestation. These results suggest that the AHI1 is a novel candidate gene for nonsyndromic RP.

\section{DISCUSSION}

In the past 2 decades, almost 200 genes have been linked to IRD. The significant genetic heterogeneity of this disease is 
a major obstacle for molecular diagnosis in clinical practice. Traditional methods of individual gene screening are difficult and unlikely to reveal the full mutation spectrum in the patient population. Tremendous efforts to develop comprehensive screening strategies have been made, including genotyping microarray, ${ }^{25}$ denaturing high-performance liquid chromatography, ${ }^{19}$ and high-resolution melting analysis. ${ }^{26}$ However, issues such as prolonged processing time and an inability to identify novel mutations persist. NGS is a high-throughput approach capable of efficiently sequencing large gene pools, and it provides large data sets. It has become a powerful tool for elucidating complete mutation profiles for heterogeneous diseases. ${ }^{27,28}$ In this study we developed a new capture panel to comprehensively screen 164 known IRD genes. Using a group of 179 unrelated patients with IRD, we successfully identified pathological mutations in 99 patients (55.3\%). Of note, among the total 124 mutation described in this study, 79 mutations were previously reported worldwide. We previously screened five patients with Bardet-Biedl syndrome for $B B S$ genes ${ }^{10}$ and five patients with Usher syndrome for USH genes $^{7}$ using the same panel; these were considered pilot studies to test the methodology and have been excluded from this study. Summarizing all these results, the detection rate was $57.7 \%(109 / 189)$. To the best of our knowledge, this is the largest investigation of genetic screening in Chinese patients with IRD. The previous studies focused only on several specific genes or screened a small cohort of patients; therefore, the contributions of causative genes to IRD in patients remain unclear. The results of this study outline a significant mutation spectrum in Chinese patients with IRD. Among the 35 genes identified in the cohort of 179 patients, the genes with the highest frequency of mutations were USH2A, EYS, and CRB1, indicating that these genes should be a priority when screening Chinese patients. This improved knowledgebase provides valuable insights and aids in genetic counseling, diagnosis, and potential therapy for patients with IRD.

Epidemiological studies assessing different populations have indicated that half of all mutations in patients with IRD are sporadic or multiplex, and, unfortunately, those cases are the ones most prevalent in the clinic. ${ }^{19,29}$ In our cohort of patients with IRD, $90 \%$ of probands displayed sporadic or autosomal recessive mutations, which makes molecular diagnosis more challenging. ${ }^{30}$ Several groups, including ours, have attempted to challenge the molecular diagnosis of simplex or autosomal recessive IRD ${ }^{19,31,32}$; however, the detection rates remained lower than $20 \%$. In this study, however, our results showed that $50 \%$ of patients with sporadic and $71 \%$ of patients with recessive IRD were successfully determined to have genetic predispositions, suggesting that targeted exome sequencing is an efficient and applicable approach for the molecular diagnosis of such patients. In addition, the mutation spectrum delineated in our cohort provides essential information for molecular diagnosis as well as for future gene therapy in Chinese patients with IRD. Notably, two unrelated patients in our cohort were identified as having RPE65 mutations, indicating a possibility of treatment with gene therapy, as several clinical trials have shown significant effects for RPE65..$^{33}$ By contrast, 80 patients had no mutations identified among the screened genes, suggesting a high possibility of novel undiscovered causes, which warrants further investigation. Most IRD-related genes are critical for physiological or developmental function of the retina. ${ }^{1}$ For instance, the dysfunction of genes involved in either of the phototransduction cascades, such as the $C R X$ and $N R L$ genes, leads to retinal dystrophy. ${ }^{34,35}$ With the aim of detecting mutations in possible novel candidate genes in a cost-effective way, a capture panel including 88 possible candidate genes and 32 retina-abundant miRNAs was designed. Most recently, mutations in the SIX6 gene, included in our panel, were linked to an abnormal proliferation-differentiation balance and optic nerve degeneration. ${ }^{36}$ However, we observed no such pathogenic mutation in the predicted candidate genes or miRNAs regions. This result might be because of true nonpathogenicity or a simply small proportion of these genes in our panel.

In general, IRD presents with high genetic and clinical heterogeneity. So far, few studies have described a genotype-phenotype correlation, mainly because of the limited effectiveness of genetic screening approaches. In this study we successfully identified disease-causing mutations in 99 patients, providing the opportunity to evaluate genotype-phenotype correlations. We investigated the relationships between a lack of visual acuity and age at disease onset, which have different causative mutations. Notably, the known IRD genes could be loosely classified into three distinct groups, showing severe, moderate, or mild visual acuity phenotypes. Trends within genotypes for patients' age at onset also were noted. However, examination of a larger cohort and identification of more mutations causing IRD are needed to help further orient our findings as genetic associations. These results demonstrated that disease severity might be gene specific, knowledge that will be of value in prognosis and future therapeutic interventions.

Interestingly, the first case of ESCS in the Chinese population and a rare case of unilateral macular coloboma with $R H O$ gene mutation were identified in this study. Patient W29-1 was diagnosed as having RP. However, a previously reported NR2E3 gene mutation was discovered in this proband; this mutation also cosegregated with phenotype in the patient's family. Therefore, we revisited the proband and observed clinical features that were in accordance with ESCS (Supplementary Figure S1 online), resulting in a revised clinical diagnosis of ESCS. Together, these results clearly illustrate the power and importance of the combination of genotype and phenotype in the molecular diagnosis of heterogeneous diseases.

For the first time, our study links mutations in the AHI1 gene to nonsyndromic RP. AHI1 mutations were first observed in patients with JBS, a developmental brain disorder with diverse symptoms in several organs, including the retina. ${ }^{37}$ The diagnostic hallmark of retinal JBS is a unique cerebellar and brainstem malformation. In our study patient W196-1, who had sporadic RP, was identified as having compound heterozygous mutations 
(c.653A>G (p.Y218C) and c.3257A>G (p.E1086G)) in AHI1 (Supplementary Figure S3 online). The mutations completely cosegregated with phenotype in all family members tested. The missense mutation Y218C was reported initially, whereas another mutation was identified as a causative mutation of JBS in previous studies. ${ }^{24}$ However, the proband showed typical RP phenotypes, without any additional manifestation of JBS. This result indicates that $A H I$ is a novel candidate gene for nonsyndromic RP. Moreover, several previous studies demonstrated that an affected gene from syndromic RP could also cause isolated retinal disease with no additional symptoms. Examples include CEP290, mutations in which can cause either JBS or Leber congenital amaurosis, ${ }^{38}$ and $C L N 3$, which is associated with Batten disease and nonsyndromic retinal degeneration. ${ }^{39}$ The novel genotype-phenotype correlations described in this study enhance our knowledge of the molecular mechanisms of retinal dystrophies.

In summary, we have developed a paneled exome sequencing methodology for the molecular diagnosis of IRD and identified 124 mutations, including 79 novel mutations, in a group of 179 Chinese patients. Furthermore, novel genotype-phenotype correlations of IRD were uncovered, and we established a novel candidate gene for nonsyndromic RP. To the best of our knowledge, this is the largest comprehensive genetic screening of Chinese IRD to date. This study expands the field of genotypephenotype correlations and the mutational spectrum of the Chinese population with IRD, increasing our understanding of molecular mechanisms of the disease and aiding the clinical diagnosis and personalized treatment of patients with IRD.

\section{SUPPLEMENTARY MATERIAL}

Supplementary material is linked to the online version of the paper at http://www.nature.com/gim

\section{ACKNOWLEDGMENTS}

We thank the patients and family members for their participation in this study. This work was supported by grants from the National Key Basic Research Program (2013CB967502 to Z.-B.J., 2011 CB504600 to J.Q.), the National Natural Science Foundation of China (81371059 to Z.-B.J.), and MOST Projects (2012YQ12008004 to F.L.), and was funded in part by a Qianjiang Scholarship (to Z.-B.J.) and grants from the Zhejiang provincial \& Ministry of Health research fund for medical sciences (to Z.-B.J.) and the Zhejiang Provincial Natural Science Foundation of China (LR13H120001 to Z.-B.J.).

\section{DISCLOSURE}

The authors declare no conflict of interest.

\section{REFERENCES}

1. Hartong DT, Berson EL, Dryja TP. Retinitis pigmentosa. Lancet 2006;368: 1795-1809.

2. Daiger SP, Sullivan LS, Bowne SJ. Genes and mutations causing retinitis pigmentosa. Clin Genet 2013;84:132-141.

3. Korf $B R$, Rehm HL. New approaches to molecular diagnosis. JAMA 2013;309:1511-1521.
4. Dixon-Salazar TJ, Silhavy JL, Udpa N, et al. Exome sequencing can improve diagnosis and alter patient management. Sci Trans/ Med 2012;4:138ra78.

5. Jin ZB, Huang XF, Lv JN, et al. SLC7A14 linked to autosomal recessive retinitis pigmentosa. Nat Commun 2014;5:3517.

6. Abu-Safieh L, Alrashed M, Anazi S, et al. Autozygome-guided exome sequencing in retinal dystrophy patients reveals pathogenetic mutations and novel candidate disease genes. Genome Res 2013;23:236-247.

7. Huang XF, Xiang P, Chen J, et al. Targeted exome sequencing identified novel USH2A mutations in Usher syndrome families. PLoS One 2013;8:e63832.

8. Wang $X$, Wang $H$, Sun V, et al. Comprehensive molecular diagnosis of 179 Leber congenital amaurosis and juvenile retinitis pigmentosa patients by targeted next generation sequencing. J Med Genet 2013;50:674-688.

9. Chen X, Zhao K, Sheng X, et al. Targeted sequencing of 179 genes associated with hereditary retinal dystrophies and 10 candidate genes identifies novel and known mutations in patients with various retinal diseases. Invest Ophthalmol Vis Sci 2013;54:2186-2197.

10. Xing DJ, Zhang HX, Huang N, et al. Comprehensive molecular diagnosis of Bardet-Biedl syndrome by high-throughput targeted exome sequencing. PLoS One 2014;9:e90599.

11. Carvill GL, Heavin SB, Yendle SC, et al. Targeted resequencing in epileptic encephalopathies identifies de novo mutations in CHD2 and SYNGAP1. Nat Genet 2013;45:825-830.

12. Hurd T, Zhou W, Jenkins $P$, et al. The retinitis pigmentosa protein RP2 interacts with polycystin 2 and regulates cilia-mediated vertebrate development. Hum Mol Genet 2010;19:4330-4344.

13. Boylan JP, Wright AF. Identification of a novel protein interacting with RPGR. Hum Mol Genet 2000;9:2085-2093.

14. Damiani D, Alexander JJ, O'Rourke JR, et al. Dicer inactivation leads to progressive functional and structural degeneration of the mouse retina. J Neurosci 2008;28:4878-4887.

15. Trimarchi JM, Stadler MB, Cepko CL. Individual retinal progenitor cells display extensive heterogeneity of gene expression. PLoS One 2008;3:e1588.

16. Livesey FJ, Young TL, Cepko CL. An analysis of the gene expression program of mammalian neural progenitor cells. Proc Natl Acad Sci USA 2004;101: 1374-1379

17. Krol J, Busskamp V, Markiewicz I, et al. Characterizing light-regulated retinal microRNAs reveals rapid turnover as a common property of neuronal microRNAs. Cell 2010;141:618-631.

18. Ryan DG, Oliveira-Fernandes $M$, Lavker RM. MicroRNAs of the mammalian eye display distinct and overlapping tissue specificity. Mol Vis 2006;12:1175-1184.

19. Jin ZB, Mandai M, Yokota T, et al. Identifying pathogenic genetic background of simplex or multiplex retinitis pigmentosa patients: a large scale mutation screening study. J Med Genet 2008;45:465-472.

20. Haider NB, Jacobson SG, Cideciyan AV, et al. Mutation of a nuclear receptor gene, NR2E3, causes enhanced S cone syndrome, a disorder of retinal cell fate. Nat Genet 2000;24:127-131.

21. Wright AF, Reddick AC, Schwartz SB, et al. Mutation analysis of NR2E3 and NRL genes in Enhanced S Cone Syndrome. Hum Mutat 2004;24:439.

22. Li S, Xiao X, Wang P, Guo X, Zhang Q. Mutation spectrum and frequency of the RHO gene in 248 Chinese families with retinitis pigmentosa. Biochem Biophys Res Commun 2010;401:42-47.

23. Ferland RJ, Eyaid W, Collura RV, et al. Abnormal cerebellar development and axonal decussation due to mutations in AHI1 in Joubert syndrome. Nat Genet 2004;36:1008-1013.

24. Kroes HY, van Zon PH, Fransen van de Putte D, et al. DNA analysis of AHI1, NPHP1 and CYCLIN D1 in Joubert syndrome patients from the Netherlands. Eur J Med Genet 2008;51:24-34.

25. Zernant J, Külm M, Dharmaraj S, et al. Genotyping microarray (disease chip) for Leber congenital amaurosis: detection of modifier alleles. Invest Ophthalmol Vis Sci 2005;46:3052-3059.

26. Simpson DA, Clark GR, Alexander S, Silvestri G, Willoughby CE. Molecular diagnosis for heterogeneous genetic diseases with targeted high-throughput DNA sequencing applied to retinitis pigmentosa. J Med Genet 2011;48: 145-151.

27. Neveling K, Collin RW, Gilissen C, et al. Next-generation genetic testing for retinitis pigmentosa. Hum Mutat 2012;33:963-972.

28. Coppieters F, Van Schil K, Bauwens M, et al. Identity-by-descent-guided mutation analysis and exome sequencing in consanguineous families reveals unusual clinical and molecular findings in retinal dystrophy. Genet Med 2014;16:671-680. 


\section{ORIGINAL RESEARCH ARTICLE}

29. Nájera C, Millán JM, Beneyto M, Prieto F. Epidemiology of retinitis pigmentosa in the Valencian community (Spain). Genet Epidemiol 1995; 12:37-46.

30. Iwanami M, Oshikawa M, Nishida T, Nakadomari S, Kato S. High prevalence of mutations in the EYS gene in Japanese patients with autosomal recessive retinitis pigmentosa. Invest Ophthalmo/ Vis Sci 2012;53:1033-1040.

31. Ávila-Fernández A, Cantalapiedra D, Aller E, et al. Mutation analysis of 272 Spanish families affected by autosomal recessive retinitis pigmentosa using a genotyping microarray. Mol Vis 2010;16:2550-2558.

32. Clark GR, Crowe P, Muszynska D, et al. Development of a diagnostic genetic test for simplex and autosomal recessive retinitis pigmentosa. Ophthalmology 2010;117:2169-77.e3.

33. Bainbridge JW, Smith AJ, Barker SS, et al. Effect of gene therapy on visual function in Leber's congenital amaurosis. N Engl J Med 2008;358: 2231-2239.

34. Freund CL, Gregory-Evans CY, Furukawa T, et al. Cone-rod dystrophy due to mutations in a novel photoreceptor-specific homeobox gene (CRX) essential for maintenance of the photoreceptor. Cell 1997;91:543-553.

35. Farjo Q, Jackson A, Pieke-Dahl S, et al. Human bZIP transcription factor gene NRL: structure, genomic sequence, and fine linkage mapping at 14q11.2 and negative mutation analysis in patients with retinal degeneration. Genomics 1997:45:395-401.

36. Iglesias Al, Springelkamp $\mathrm{H}$, van der Linde $\mathrm{H}$, et al. Exome sequencing and functional analyses suggest that SIX6 is a gene involved in an altered
HUANG et al | Inherited retinal dystrophy revealed by next-generation sequencing

proliferation-differentiation balance early in life and optic nerve degeneration at old age. Hum Mol Genet 2014;23:1320-1332.

37. Romani M, Micalizzi A, Valente EM. Joubert syndrome: congenital cerebellar ataxia with the molar tooth. Lancet Neuro/ 2013;12:894-905.

38. den Hollander Al, Koenekoop RK, Yzer S, et al. Mutations in the CEP290 (NPHP6) gene are a frequent cause of Leber congenital amaurosis. Am J Hum Genet 2006;79:556-561.

39. Wang $\mathrm{F}$, Wang $\mathrm{H}$, Tuan $\mathrm{HF}$, et al. Next generation sequencing-based molecular diagnosis of retinitis pigmentosa: identification of a novel genotype-phenotype correlation and clinical refinements. Hum Genet 2014;133:331-345.

This work is licensed under a Creative Commons
Attribution-NonCommercial-NoDerivs $\quad 3.0$

Unported License. The images or other third party material in this article are included in the article's Creative Commons license, unless indicated otherwise in the credit line; if the material is not included under the Creative Commons license, users will need to obtain permission from the license holder to reproduce the material. To view a copy of this license, visit http://creativecommons.org/licenses/by-nc-nd/3.0/ 